\title{
HOMOLOGICAL ALGEBRA AND SET THEORY
}

BY

\author{
PAUL C. EKLOF( $\left({ }^{1}\right)$
}

\begin{abstract}
Assuming the Axiom of Constructibility, necessary and sufficient conditions are given for the vanishing of Ext $t_{\Lambda}^{\prime}$ for rings $\Lambda$ of global dimension 1. Using Martin's Axiom, the necessity of these conditions is shown not to be a theorem of ZFC. Applications are given to abelian group theory, including a partial solution (assuming $\mathrm{V}=\mathrm{L}$ ) to a problem of Baer on the splitting of abelian groups. Some independence results in abelian group theory are also proved.
\end{abstract}

Introduction. In a remarkable recent paper [22], S. Shelah proved that Whitehead's problem is unsolvable in ordinary set theory (Zermelo-Frankel set theory with the Axiom of Choice). More precisely, he proved that two different answers to the problem are obtained when set theory is extended by adding two different axioms: the Axiom of Constructibility and Martin's Axiom. (See Theorem 4.1.) Shelah's arguments were specific to Whitehead's problem and nonhomological in character. In this paper we extend Shelah's techniques in order to obtain some general results about the vanishing of Ext $_{\Lambda}^{1}$ (for modules over a ring $\Lambda$ of global dimension 1) in different models of set theory, and we apply them to some specific problems in abelian group theory.

Assuming the Axiom of Constructibility we obtain necessary and sufficient conditions for the vanishing of $\operatorname{Ext}_{\Lambda}^{1}$ (Theorems 1.2 and 1.5). To state the result in a special case: if $C$ and $A$ are abelian groups of cardinality $N_{1}$, $\operatorname{Ext}(C, A)=0$ if and only if $C$ is the union of an increasing chain of countable subgroups $\left\{C_{\nu} \mid \nu<N_{1}\right\}$ such that the chain is smooth (i.e., $C_{\sigma}=$ $\cup_{\nu<\sigma} C_{\nu}$ for all limit ordinals $\left.\sigma\right)$ and satisfies $\operatorname{Ext}\left(C_{0}, A\right)=0$ and $\operatorname{Ext}\left(C_{p+1} / C_{\nu}, A\right)=0$ for all $\nu<\kappa_{1}$. Although the sufficiency of this condition is an easy consequence of classical homological algebra (see Theorem 1.2), the proof of necessity makes use of the Axiom of Constructibility (Theorem 1.5). The necessity of this condition is not provable in ordinary set theory; in particular the condition is not necessary when Martin's Axiom is assumed (\$3).

Received by the editors March 3, 1975.

AMS (MOS) subject classifications (1970). Primary 02K05, 18G15, $20 \mathrm{~K} 35$.

(')Research supported by NSF GP43910.

- American Mathematical Society 1977 
For those who are not interested in algebra in the constructible universe, the result cited above as well as the other results of $\S \S 1$ and 2 are still of interest as consistency results. For example, with respect to the result cited above, since the Axiom of Constructibility is consistent with ordinary set theory, we can conclude from this result that no condition weaker than the one given can be proven in ordinary set theory to be sufficient to imply that $\operatorname{Ext}(C, A)=0$ (although weaker conditions might suffice in some models of ordinary set theory).

One of the applications is to the problem of splitting abelian groups (\$2). Assuming the Axiom of Constructibility, we give a necessary and sufficient condition on pairs $(G, T)$ with $G$ torsion-free of cardinality $\kappa_{1}$ and $T$ torsion such that $\operatorname{Ext}(G, T)=0$. This condition is more complicated than Baer's criterion for pairs $(G, T)$ where $G$ is countable; we analyze the difference more precisely using infinitary logic.

We also give some independence results for homological problems (\$4). To cite one example related to Baer's problem: the problem of whether for torsion-free groups $G$ of cardinality $\aleph_{1}$, the vanishing of $\operatorname{Ext}(G, T)$ for all countable torsion groups $T$ implies $G$ is free is unsolvable in ordinary set theory (Theorem 4.6).

We begin by reviewing some notions and results from set theory.

0 . Set-theoretic preliminaries. We shall always identify an ordinal $\mu$ with the set of ordinals less than $\mu: \mu=\{\nu \mid \nu<\mu\}$. A cardinal is identified with an initial ordinal; thus $\omega_{\alpha}$ is identified with $\kappa_{\alpha}$. If $A$ is a set, $\overline{\bar{A}}$ denotes the cardinality of $A$.

If $\sigma$ is a limit ordinal, the cofinality of $\sigma$ is the smallest ordinal $\kappa$ such that there is a strictly increasing function $f: \kappa \rightarrow \sigma$ such that $\sup \{f(\nu): \nu<\kappa\}=$ $\sigma$. We write $\operatorname{cf}(\sigma)=\kappa$; it may be proved that $\kappa$ is an infinite cardinal $<\sigma$. A cardinal $\kappa$ is said to be regular if $\operatorname{cf}(\kappa)=\kappa$, otherwise $\kappa$ is singular. For example, any successor cardinal is regular.

Let $\kappa$ and $\sigma$ be limit ordinals. A function $f: \kappa \rightarrow \sigma$ is called normal if $f$ is continuous (i.e., $f(\mu)=\sup \{f(\nu) \mid \nu<\mu\}$ for every limit ordinal $\mu<\kappa)$ and strictly increasing. A subset $S$ of $\sigma$ is stationary in $\sigma$ if $S$ meets the range of every normal function $f: \operatorname{cf}(\sigma) \rightarrow \sigma$. Equivalently, $S$ is stationary in $\sigma$ if and only if $S$ meets every closed cofinal subset of $\sigma$. For example, $S_{1}=$ the set of all limit ordinals less than $\sigma$, and $S_{2}=$ the set of all limit ordinals of cofinality $\omega$ less than $\sigma$ are stationary in $\sigma$, provided $\sigma$ has cofinality $>\omega$.

Let $C$ be a set, $\kappa$ a cardinal. Let $\mathscr{P}(C)$ denote the set of subsets of $C$. Adapting terminology from Rotman [20] we define: a $\kappa$-filtration of $C$ is a function $\Delta: \kappa \rightarrow \mathscr{P}(C)$ such that:

(i) for all $\nu<\kappa, \overline{\Delta(\nu)}<\kappa$; 
(ii) for all $\nu<\kappa, \Delta(\nu) \subseteq \Delta(\nu+1)$;

(iii) (smoothness) if $\sigma$ is a limit ordinal $<\kappa$, then $\Delta(\sigma)=\cup_{\nu<\sigma} \Delta(\nu)$; and

(iv) $C=\cup_{\nu<k} \Delta(\nu)$.

If $C$ is a module. we require $\Delta(\nu)$ to be a submodule, and denote $\Delta(\nu+1) / \Delta(\nu)$ by $\bar{\Delta}(\nu)$. For regular $\kappa, C$ has a $\kappa$-filtration if and only if $\overline{\bar{C}}<\kappa$.

Let ZFC denote the axioms of Zermelo-Frankel set theory plus the Axiom of Choice. We shall be interested in some axioms which can consistently be added to ZFC. The first of these is Gödel's Axiom of Constructibility, denoted V = L. Gödel proved the following [11].

0.1 THEOREM. If ZFC is consistent, then $\mathrm{ZFC}+\mathrm{V}=\mathrm{L}$ is consistent.

In what follows we make use of two consequences of Gödel's axiom discovered by $R$. Jensen [15]. (The parenthetical notation $(V=L)$ means that the result stated is a theorem of $\mathrm{ZFC}+\mathrm{V}=\mathrm{L}$.)

0.2 THEOREM $(\mathrm{V}=\mathrm{L})$. Let $\kappa$ be a regular uncountable cardinal. Let $X$ and $Y$ be sets of cardinality $\kappa$ and let $\Delta_{X}$ and $\Delta_{Y}$ be $\kappa$-filtrations of $X$ and $Y$ respectively. Let $S$ be a stationary subset of $\kappa$. Then there is a sequence of functions $\left\{f_{\nu}: \Delta_{X}(\nu) \rightarrow \Delta_{Y}(\nu) \mid \nu \in S\right\}$ indexed by $S$ such that for every function $g: X \rightarrow Y$ there exists $\nu \in S$ such that $g$ restricted to $\Delta_{X}(\nu)$ equals $f_{\nu}$.

REMARK. We have stated Jensen's result (usually expressed as $\nabla_{\kappa}(S)$ ) in the form most convenient for our use. This version is easily derived from [15, Lemma 6.5] with the help of the observation that there is a closed cofinal set $C$ of $\nu<\kappa$ with the property that $g\left(\Delta_{X}(\nu)\right) \subseteq \Delta_{Y}(\nu)$; for $\nu$ in $C, g$ restricted to $\Delta_{X}(\nu)$ equals $g \cap\left(\Delta_{X}(\nu) \times \Delta_{Y}(\nu)\right)$.

The second result of Jensen which we need (for the proof of Theorem 2.5) is the following [15, Theorem 6.1]. (For the definition of a weakly compact cardinal see, for example, Drake [5]. Here we simply note that any successor cardinal is not weakly compact.)

0.3 THEOREM $(\mathrm{V}=\mathrm{L})$. For any regular cardinal $\kappa$ which is not weakly compact there is a subset $S$ of $\kappa$ such that:

(1) $S$ is stationary in $\kappa$;

(2) every element of $S$ is a limit ordinal of cofinality $\omega$; and

(3) for every limit ordinal $\sigma<\kappa, S \cap \sigma$ is not stationary in $\sigma$.

Another axiom whose consequences we consider is Martin's Axiom, denoted MA. This axiom is of interest in conjunction with the denial of the Continuum Hypothesis, denoted $\sim \mathrm{CH}$. The following was proved by Solovay and Tenenbaum [24].

0.4 THEOREM. If ZFC is consistent, then $\mathrm{ZFC}+\mathrm{MA}+\sim \mathrm{CH}$ is consistent. 
We state a special case of Martin's Axiom in the form of a theorem.

0.5 THEOREM (MA $+\sim \mathrm{CH})$. Let $A$ and $B$ be sets of cardinality $<2^{\mathrm{N}_{0}}$. Let $P$ be a family of functions with the following properties:

(1) every $f$ in $P$ is a function from a subset of $A$ to $B$;

(2) for every $a \in A$ and every $f \in P$, there exists a function $f^{\prime}$ in $P$ such that $a$ is in the domain of $f^{\prime}$ and $f \subseteq f^{\prime}$; and

(3) for every uncountable subset $P^{\prime}$ of $P$, there exist functions $f_{1}, f_{2}$ in $P^{\prime}$ and $f_{3}$ in $P$ such that $f_{1} \neq f_{2}$ and $f_{3}$ extends both $f_{1}$ and $f_{2}$.

Then there exists a function $g: A \rightarrow B$ such that for every finite subset $X$ of $A, g$ restricted to $X$ is the restriction of a member of $P$.

1. The vanishing of Ext in the constructible universe. Throughout this section $\Lambda$ will denote a ring of left global dimension $<1$ (i.e., $\operatorname{Ext}_{\Lambda}^{2}(C, A)=0$ for all left $\Lambda$-modules $C$ and $A$ ). Recall the following fact which we state as a lemma. (From now on, all modules are left $A$-modules.)

1.1 LeMma. If $A$ and $C$ are $\Lambda$-modules such that $\operatorname{Ext}_{\Lambda}^{1}(C, A)=0$, then for all submodules $C^{\prime}$ of $C, \operatorname{Ext}_{\Lambda}^{1}\left(C^{\prime}, A\right)=0$.

Proof. The inclusion of $C^{\prime}$ into $C$ induces a map

$$
\operatorname{Ext}_{\Lambda}^{1}(C, A) \rightarrow \operatorname{Ext}_{\Lambda}^{1}\left(C^{\prime}, A\right) \rightarrow \operatorname{Ext}_{\Lambda}^{2}\left(C / C^{\prime}, A\right)=0
$$

(see [3]). Since $\operatorname{Ext}_{\Lambda}^{1}(C, A)=0$, surjectivity implies $\operatorname{Ext}_{\Lambda}^{1}\left(C^{\prime}, A\right)=0$.

Throughout the paper $\kappa$ will denote a regular uncountable cardinal $>\overline{\bar{\Lambda}}$ (but see Remark 1.9(2)). Given a $\Lambda$-module $A$, we are interested in finding necessary and sufficient conditions on $C$ such that $\operatorname{Ext}_{\Lambda}^{1}(C, A)=0$. We are interested in conditions involving the submodules of $C$ of smaller cardinality. Let $\overline{\bar{C}}=\kappa$. We begin with a necessary condition.

Recall that $\operatorname{Ext}_{\Lambda}^{1}(C, A)=0$ if and only if for every surjective homomorphism $\pi: B \rightarrow C$ such that $\operatorname{ker} \pi=A$, there is a splitting for $\pi$, i.e., a homomorphism $\rho: C \rightarrow B$ such that $\pi \circ \rho=1_{C}$. (See [3, Theorem 1.1, p. 290].)

1.2 Theorem. Let $C$ and $A$ be $\Lambda$-modules and let $\kappa=\overline{\bar{C}}$. Suppose that there is a $\kappa$-filtration $\Delta_{C}$ of $C$ satisfying:

(1) $\operatorname{Ext}_{\Lambda}^{1}\left(\Delta_{C}(0), A\right)=0$;

and for all $\nu<\kappa$ :

(2) $\operatorname{Ext}_{\Lambda}^{1}\left(\bar{\Delta}_{C}(\nu), A\right)=0$.

Then $\operatorname{Ext}_{\Lambda}^{1}(C, A)=0$.

Proof. Consider a short exact sequence $0 \rightarrow A \rightarrow^{\prime} B \rightarrow^{\pi} C \rightarrow 0$. We shall define by transfinite induction an increasing chain of homomorphisms $\rho_{\nu}: \Delta_{C}(\nu) \rightarrow B$ indexed by $\kappa$ such that for all $\nu<\kappa, \pi \rho_{\nu}$ is the identity on 
$\Delta_{C}(\nu)$. If $\rho$ is the union of the chain then obviously $\rho$ will be a splitting for $\pi$. Suppose that $\rho_{\nu}$ has been defined for all $\nu<\mu$. If $\mu$ is a limit ordinal, let $\rho_{\mu}$ be the union of the $p_{\nu}$ for $\nu<\mu$. If $\mu$ is of the form $\nu+1$ first observe that by induction we may assume that $\operatorname{Ext}_{\Lambda}^{1}\left(\Delta_{C}(\nu), A\right)=0$. Hence

$$
\operatorname{Ext}_{\Lambda}^{1}\left(\Delta_{C}(\nu+1), A\right)=0 \text { since } \operatorname{Ext}_{\Lambda}^{1}\left(\bar{\Delta}_{C}(\nu), A\right)=0 .
$$

Also, since $\left.\operatorname{Ext}_{\Lambda}^{1} \bar{\Delta}_{C}(\nu), A\right)=0$, the map $\operatorname{Hom}\left(\Delta_{C}(\mu), A\right) \rightarrow \operatorname{Hom}\left(\Delta_{C}(\nu), A\right)$ induced by inclusion is surjective. Let $\sigma: \Delta_{C}(\mu) \rightarrow B$ be an arbitrary splitting for $\pi \mid \pi^{-1}\left(\Delta_{C}(\mu)\right) ; \sigma$ exists since $\operatorname{Ext}_{\Lambda}^{1}\left(\Delta_{C}(\mu), A\right)=0$. Let $\theta: \Delta_{C}(\nu) \rightarrow A$ be the unique homomorphism such that $i \theta=\rho_{\nu}-\left(\sigma \mid \Delta_{C}(\nu)\right)$. By the observation above, $\theta$ extends to a homomorphism $\Psi: \Delta_{C}(\mu) \rightarrow A$. If we let $\rho_{v+1}=\sigma+$ $\imath \Psi$, then $\rho_{v+1}$ has the desired properties.

We shall now proceed to prove that the converse of Theorem 1.2 is a theorem of $\mathrm{ZFC}+\mathrm{V}=\mathrm{L}$ (when $\overline{\bar{C}}$ is regular and $\overline{\bar{A}}<\overline{\bar{C}}$ ). In $\$ 3$ we shall show by counterexample that the converse is not provable in ZFC.

1.3 Lemma. Let $C_{0}$ be a submodule of $C_{1}$ such that $\operatorname{Ext}_{\Lambda}^{1}\left(C_{1}, A\right)=0$ but $\operatorname{Ext}_{\Lambda}^{1}\left(C_{1} / C_{0}, A\right) \neq 0$. Let $E: 0 \rightarrow A \rightarrow{ }^{i_{0}} B_{0} \rightarrow^{\pi_{0}} C_{0} \rightarrow 0$ be a short exact sequence and let $\rho_{0}: C_{0} \rightarrow B_{0}$ be a splitting for $\pi_{0}$. Then there is an extension $B_{1}$ of $B_{0}$ and a commutative diagram

$$
\begin{gathered}
E_{0}: 0 \longrightarrow A \stackrel{\iota_{0}}{\longrightarrow} B_{0} \stackrel{\pi_{0}}{\longrightarrow} C_{0} \longrightarrow 0 \\
\begin{array}{c}
\text { ก। } \\
\text { ก। }
\end{array} \\
E_{1}: 0 \longrightarrow A \stackrel{\iota_{1}}{\longrightarrow} B_{1} \stackrel{\pi_{1}}{\longrightarrow} C_{1} \longrightarrow 0
\end{gathered}
$$

with exact rows such that there is no splitting for $\pi_{1}$ which extends $\rho_{0}$.

Proof. Since $E_{0}$ splits, it suffices to prove the lemma in the case that $B_{0}=C_{0} \oplus A, \iota_{0}$ is inclusion, $\pi_{0}$ is projection onto the first factor and $\rho_{0}$ is the canonical embedding taking $c \in C_{0}$ to $(c, 0)$ in $B_{0}$. From the long exact sequence for Ext (see [3]) we obtain an exact sequence

$$
\operatorname{Hom}\left(C_{1}, A\right) \stackrel{\alpha^{*}}{\rightarrow} \operatorname{Hom}\left(C_{0}, A\right) \rightarrow \operatorname{Ext}_{\Lambda}^{1}\left(C_{1} / C_{0}, A\right) \rightarrow \operatorname{Ext}_{\Lambda}^{1}\left(C_{1}, A\right)
$$

where $\alpha^{*}$ is induced by the inclusion $\alpha: C_{0} \subseteq C_{1}$. It follows from the hypotheses that $\alpha^{*}$ is not onto, so let $\Psi: C_{0} \rightarrow A$ be a homomorphism which is not in the image of $\alpha^{*}$. Let $B_{1}=C_{1} \oplus A$ and define an embedding $\theta: B_{0} \rightarrow B_{1}$ by $\theta(c, a)=(c, a+\Psi(c))$. Then there is a commutative diagram 


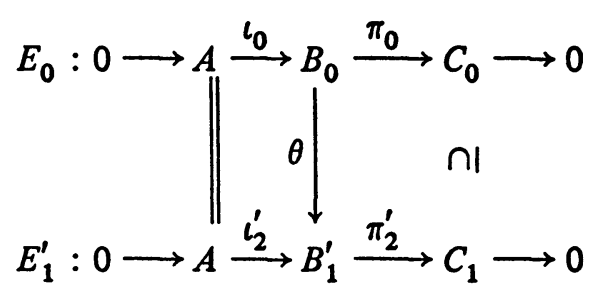

where $l_{1}^{\prime}$ and $\pi_{2}^{\prime}$ are inclusion and projection respectively. Suppose that $\rho_{1}: C_{1} \rightarrow B_{1}$ is a splitting homomorphism for $\pi_{2}^{\prime}$ such that $\rho_{2} \mid C_{0}=\theta \circ \rho_{0}$. Let $\varphi=p \circ \rho_{1}: C_{1} \rightarrow A$ where $p: B_{1} \rightarrow A$ is projection on the second factor. Then for any $c \in C_{0}, \varphi(c)=p \rho_{1}(c)=p \theta \rho_{0}(c)=\Psi(c)$; so $\varphi$ is an extension of $\Psi$, which is a contradiction. We complete the proof by using a standard set-theoretic argument to obtain a diagram (1.3.1) isomorphic to (1.3.2).

The heart of the proof of the converse of Theorem 1.2 (assuming V $=\mathrm{L}$ ) is contained in the following result. (The key idea of using Theorem 0.2 comes from Shelah [22].)

1.4 Lemma $(V=\mathrm{L})$. Let $\kappa$ be a regular uncountable cardinal $>\overline{\bar{\Lambda}}$, and let $C$ and $A$ be $\Lambda$-modules such that $\overline{\bar{C}}=\kappa$ and $\overline{\bar{A}}<\kappa$. If there is a $\kappa$-filtration $\Delta_{C}$ of $C$ such that $S=\left\{\nu<\kappa \mid \operatorname{Ext}_{\Lambda}^{1}\left(\bar{\Delta}_{C}(\nu), A\right) \neq 0\right\}$ is stationary in $\kappa$, then $\operatorname{Ext}_{\Lambda}^{1}(C, A) \neq 0$.

Proof. By Lemma 1.1 we may assume $\operatorname{Ext}_{\Lambda}^{1}\left(\Delta_{C}(\nu), A\right)=0$ for all $\nu<\kappa$. We shall construct a nonsplitting short exact sequence $E: 0 \rightarrow A \rightarrow^{\mathrm{l}} B \rightarrow^{\pi} C$ $\rightarrow 0$ as the union of a chain of short exact sequences $E_{v}: 0 \rightarrow A \rightarrow \rightarrow^{3} B_{\nu}$ $\rightarrow \pi_{C}(\nu) \rightarrow 0$. These sequences will be constructed by transfinite induction so that whenever $\mu<\nu$ there is a commutative diagram

$$
\begin{aligned}
& E_{\mu}: 0 \longrightarrow A \stackrel{\iota_{\mu}}{\longrightarrow} B_{\mu} \stackrel{\pi_{\mu}}{\longrightarrow} \Delta_{C}(\mu) \longrightarrow 0 \\
& \begin{array}{l}
\text { In } \\
E_{\nu}: 0 \longrightarrow A \stackrel{\nu_{\nu}}{\longrightarrow} B_{\nu} \stackrel{\pi_{\nu}}{\longrightarrow} \Delta_{C}(\nu) \longrightarrow 0 .
\end{array}
\end{aligned}
$$

Moreover, $E_{\nu}$ will be defined so that the underlying set of $B_{\nu}$ is $\Delta_{C}(\nu) \times A$. Choose a $k$-filtration $\Delta_{A}$ of $A$. Let $\left\{f_{\nu}: \Delta_{C}(\nu) \rightarrow \Delta_{C}(\nu) \times \Delta_{A}(\nu) \mid \nu \in S\right\}$ be the sequence of functicns given by Theorem 0.2 , with $X=C, Y=C \times A$, $\Delta_{X}=\Delta_{C}$, and $\Delta_{Y}(\nu)=\Delta_{C}(\nu) \times \Delta_{A}(\nu)$. Let $E_{0}$ be arbitrary. Suppose that $E_{\mu}$ has been defined for all $\mu<\tau$. If $\tau$ is a limit ordinal, let $E_{\tau}$ be the union of the $E_{\mu}, \mu<\tau$. If $\tau$ is a successor ordinal, say $\tau=\nu+1$, and either $\nu \notin S$ or $f_{\nu}$ 
is not a splitting homomorphism for $\pi_{\nu}$, let $E_{\tau}$ be an arbitrary extension of $E_{v}$ such that (1.4.1) commutes. If $\nu \in S$ and $f_{\nu}$ is a splitting homomorphism for $\pi_{v}$, apply Lemma 1.3 , to obtain an extension $E_{\tau}$ of $E_{v}$ such that there is no splitting homomorphism for $\pi_{\tau}$ which extends $f_{v}$. By cardinality considerations we can choose $E_{\tau}$ so that the underlying set of $B_{\tau}$ is $\Delta_{C}(\tau) \times A$. Now let $E: 0 \rightarrow A \rightarrow^{6} B \rightarrow^{\pi} C \rightarrow 0$ be the union of the $E_{\mu}, \mu<\kappa$. Then the underlying set of $B$ is $C \times A$. Suppose there is a splitting homomorphism $g: C \rightarrow B$ for $\pi: B \rightarrow C$. By choice of $\left\{f_{\nu} \mid \nu \in S\right\}$ there exists $\nu \in S$ such that $g \mid \Delta_{C}(\nu)=f_{\nu}$. Clearly $g \mid \Delta_{C}(\nu)=f_{v}$ is a splitting homomorphism for $\pi_{v}$ which extends to a splitting homomorphism $g \mid \Delta_{c}(\nu+1)$ for $\pi_{v+1}$. But this contradicts the construction of $E_{\nu+1}$. Hence $E$ does not split, and the theorem is proved.

The following is the promised converse of Theorem 1.2. (The exposition of the proof has been simplified from an earlier version by a trick we learned from the proof of Lemma 1 of Mekler [19].)

1.5 THEOREM $(\mathrm{V}=\mathrm{L})$. Let $\kappa$ be a regular uncountable cardinal $>\overline{\bar{\Lambda}}$. Let $C$ and $A$ be $\Lambda$-modules such that $\overline{\bar{C}}=\kappa$ and $\overline{\bar{A}}<\kappa$. If $\operatorname{Ext}_{\Lambda}^{1}(C, A)=0$ then there is a $k$-filtration $\Delta_{C}$ of $C$ such that

and for all $\nu<\kappa$ :

$$
\operatorname{Ext}^{1}\left(\Delta_{C}(0), A\right)=0
$$

$$
\operatorname{Ext}_{\Lambda}^{1}\left(\bar{\Delta}_{C}(\nu), A\right)=0 .
$$

In fact if $\Gamma_{C}$ is any $k$-filtration of $C$, then we can take $\Delta_{C}$ to be $\Gamma_{C} \circ f$ for some normal function $f: \kappa \rightarrow \kappa$.

Proof. Given a $\kappa$-filtration $\Gamma_{C}$ of $C$, let

$$
E=\left\{\nu<\kappa: \operatorname{Ext}_{\Lambda}^{1}\left(\Gamma_{C}(\mu) / \Gamma_{C}(\nu), A\right) \neq 0 \text { for some } \nu<\mu<\kappa\right\} .
$$

Let $g: \kappa \rightarrow \kappa$ be a normal function such that for all $\nu<\kappa$, if $g(\nu) \in E$ then $\operatorname{Ext}_{\Lambda}^{1}\left(\Gamma_{C}(g(\nu+1)) / \Gamma_{C}(g(\nu)), A\right) \neq 0$; i.e., if we let $\Phi_{C}=\Gamma_{C} \circ g$ and $S=\{\nu$ $\left.<\kappa \mid \operatorname{Ext}_{\Lambda}^{1}\left(\bar{\Phi}_{C}(\nu), A\right) \neq 0\right\}$, then $\nu \notin S$ implies $g(\nu) \notin E$. By hypothesis and Lemma $1.4, S$ is not stationary in $\kappa$. Hence there is a normal function $h: \kappa \rightarrow \kappa$ whose range does not meet $S$. If we let $f=g \circ h$ and $\Delta_{C}=\Gamma_{C} \circ f$ then $\Delta_{C}$ has the desired properties. (Note that (2) holds because $h(\nu) \notin S$ implies $f(v) \notin E$; and (1) holds by Lemma 1.1.)

In case $\kappa$ is a compact cardinal conditions (apparently) weaker than those of Theorem 1.4 will suffice to prove $\operatorname{Ext}_{\Lambda}^{1}(C, A)=0$. The following has been proved by Mekler [18].

1.6 THEOREM (MEKLER). Let $\kappa$ be a weakly compact (resp. strongly compact) cardinal $>\bar{\Lambda}$ and let $C$ and $A$ be $\Lambda$-modules such that $\overline{\bar{C}}=\kappa$ (resp. $\overline{\bar{C}}$ is 
arbitrary) and $A$ is a direct sum of modules each of cardinality $<\kappa$. Suppose that $\operatorname{Ext}_{\Lambda}^{1}\left(C^{\prime}, A\right)=0$ for all submodules $C^{\prime}$ of $C$ of cardinality $<\kappa$. Then $\operatorname{Ext}_{\Lambda}^{1}\left(C^{\prime}, A\right)=0$.

1.7 REMARKS. (1) Although the definition of weakly compact conventionally includes the requirement that the cardinal be strongly inaccessible, this assumption is not used in the above theorem. Hence it follows from results of Boos [2] and Kunen [17] that there is a model of ZFC with a regular cardinal $\kappa$ which is less than $2^{\kappa_{0}}$ and satisfies the property given in Theorem 1.6 for modules $C$ of cardinality $\kappa$. (Of course $2^{\kappa_{0}}$ is very large in this model.)

(2) It follows from Theorem 2.5 that, assuming $\mathrm{V}=\mathrm{L}$, Theorem 1.6 does not hold for any regular $\kappa$ which is not weakly compact.

The following lemma will be useful in $\$ 3$ in seeing that modules constructed there do not satisfy conditions (1) and (2) of Theorem 1.5. (Note that the lemma is proved in $\mathrm{ZFC}$.)

1.8 LemMA. Let $C$ and $A$ be $\Lambda$-modules and let $\kappa=\overline{\bar{C}}$ be regular. Suppose there is a $\kappa$-filtration $\Delta_{C}$ such that $S=\left\{\nu<\kappa \mid \operatorname{Ext}_{\Lambda}^{1}(\bar{\Delta}(\nu), A) \neq 0\right\}$ is stationary in $\kappa$. Then $C$ does not have a $\kappa$-filtration which satisfies (1) and (2) of Theorem 1.5 .

Proof. Let $\Gamma$ be any $\kappa$-filtration of $C$. We shall prove that $\Gamma$ does not satisfy (2) of Theorem 1.5. We claim that there is a normal function $f: \kappa \rightarrow \kappa$ such that for all $\nu<\kappa, \Gamma(f(\nu))=\Delta(f(\nu))$. If $f(\nu)$ has been defined for all $\mu<\nu$, and $\nu$ is a limit ordinal let $f(\nu)=\sup \{f(\mu) \mid \mu<\nu\}$; if $\nu=\mu+1$ for some $\mu$, choose by induction ordinals $\sigma_{n}>\mu$ such that for all $k<\omega$,

$$
\Gamma\left(\sigma_{2 k}\right) \subseteq \Delta\left(\sigma_{2 k+1}\right) \subseteq \Gamma\left(\sigma_{2 k+2}\right) \text {. }
$$

(This is possible since $\kappa$ is regular.) Let $f(\nu)=\sup \left\{\sigma_{n} \mid n<\omega\right\}$. Then it follows from the smoothness of $\Delta$ and $\Gamma$ that $\Gamma(f(\nu))=\Delta(f(\nu))$.

Now since $S$ is stationary in $\kappa$ there exists $\nu_{0}<\kappa$ such that $f\left(\nu_{0}\right) \in S$ i.e., $\operatorname{Ext}_{\Lambda}^{1}\left(\bar{\Delta}\left(f\left(\nu_{0}\right)\right), A\right) \neq 0$. By Lemma 1.1,

$$
0 \neq \operatorname{Ext}_{\Lambda}^{1}\left(\Delta\left(f\left(\nu_{0}+1\right)\right) / \Delta\left(f\left(\nu_{0}\right)\right), A\right)=\operatorname{Ext}_{\Lambda}^{1}\left(\Gamma\left(f\left(\nu_{0}+1\right)\right) / \Gamma\left(f\left(\nu_{0}\right)\right), A\right) .
$$

But if $\Gamma$ satisfies $1.5(2)$ then it follows that $\operatorname{Ext}_{\Lambda}^{1}(\Gamma(\mu) / \Gamma(\nu), A)=0$ whenever $\nu<\mu<\kappa$. (Proof by induction on $\mu$ : apply the proof of Theorem 1.2 to the filtration $\{\Gamma(\tau) / \Gamma(\nu): \nu<\tau<\mu\}$ of $\Gamma(\mu) / \Gamma(\nu)$.) Hence we conclude that $\Gamma$ does not satisfy (2).

1.9 REMARKS. (1) Lemma 1.4 is useful in constructing examples of modules $C$ such that $\operatorname{Ext}_{\Lambda}^{1}(C, A) \neq 0$ : we construct $C$ as the union of a smooth chain $\cup_{\nu<\kappa} C_{\nu}$ of submodules of cardinality $<\kappa$ such that the filtration $\Delta_{C}(\nu)=C_{\nu}$ satisfies $\left\{\nu<\kappa \mid \operatorname{Ext}_{\Lambda}^{1}\left(\bar{\Delta}_{C}(\nu), A\right) \neq 0\right\}$ is stationary in $\kappa$. (See for example the proof of Theorem 2.4.) 
(2) If $\kappa<\overline{\bar{\Lambda}}$ (but still regular and uncountable) Theorem 1.5 still holds if $\overline{\bar{C}}=\kappa$ is interpreted to mean that $C$ is generated by $\kappa$ elements and if we require that the cardinality of $A$ is strictly less than $\kappa$. In this case a $\kappa$-filtration $\Delta_{C}$ of $C$ is defined to be a $\kappa$-filtration of a set of generators for $C$.

2. Splitting abelian groups. One of the most basic and difficult problems in the theory of abelian groups is the determination of when an abelian group splits, i.e., is the direct sum of its torsion part and a torsion-free group. Baer [1] posed the problem of describing the pairs $(G, T)$ of torsion-free and torsion abelian groups, respectively, such that $A$ splits whenever the torsion part, $A_{t}$, of $A$ is isomorphic to $T$ and $A / A_{t}$ is isomorphic to $G$. In modern terminology this problem is expressed as describing the pairs $(G, T)$ such that $\operatorname{Ext}_{\mathrm{Z}}^{1}(G, T)=0$ (cf. [8, Problem 31]; also [10, Problem 80]). Baer solved the problem for the case when $G$ is countable. Let us recall Baer's criterion. (From now on, "group" will mean abelian group. Also we will write $\operatorname{Ext}(G, T)$ instead of $\operatorname{Ext}_{\mathbf{z}}^{1}(G, T)$.) If $p$ is a prime we say that $G$ is almost $p$-infinite if there is a pure subgroup $S$ of $G$ of finite rank such that $G / S$ contains a nonzero $p$-divisible subgroup (i.e., a subgroup $H$ such that $H=p H$ $\neq 0$ ). If $P$ is an (infinite) set of primes say that $G$ is almost $P$-infinite if there exists a pure subgroup $S$ of $G$ of finite rank such that there is a nonzero element of $G / S$ which is divisible by $p$ for all $p$ in $P$. If $T$ is a torsion group let $T_{p}$ denote the $p$-primary part of $T$ i.e., $T_{p}=\left\{x \in T \mid p^{n} x=0\right.$ for some $\left.n\right\}$. Let us say that a torsion-free group $G$ is $T$-admissible, if

(i) for all $p$ such that $T_{p}$ is unbounded, $G$ is not almost $p$-infinite; and

(ii) if $P=\left\{p \mid T_{p} \neq 0\right\}$ is infinite, then $G$ is not almost $P$-infinite. Recall that $T$ is reduced if it does not contain a nonzero divisible subgroup [9, p. 100].

2.1 THeOREM (BAER). Let $T$ be a reduced torsion group and $G$ a torsion-free group. If $\operatorname{Ext}(G, T)=0$ then $G$ is $T$-admissible. If $G$ is countable and $G$ is $T$-admissible then $\operatorname{Ext}(G, T)=0$.

An easy consequence of Baer's criterion is that for countable $G$ and fixed $T$ the property $\operatorname{Ext}(G, T)=0$ is expressible as a sentence of the infinitary language $L_{\omega_{1} \omega}$ of group theory. First let us recall the definitions from infinitary logic. If $\lambda$ and $\kappa$ are infinite cardinals, $L_{\lambda \kappa}$ is the class of formulas obtained by closing the class of atomic formulas, i.e., formulas of the forms $x+y=z$ or $x=0$, under the operations of negation, existential quantification over sets of variables of cardinality $<\kappa$, and conjunction of sets of formulas of cardinality $<\kappa . L_{\infty \kappa}$ is the union of the classes $L_{\lambda \kappa}$ for all cardinals $\lambda$, and $L_{\infty \infty \infty}$ is the union of the classes $L_{\infty}$ for all cardinals $\kappa$. For more details see [4], [5] or [16]. 
2.2 CoRollary. For any reduced torsion group $T$ there is a sentence $\varphi_{T}$ of $L_{\omega, \omega}$ such that for any countable torsion-free group $G, \operatorname{Ext}(G, T)=0$ if and only if $G$ satisfies $\varphi_{T}$.

Proof. Let $S=\left\{p \mid T_{p}\right.$ is unbounded $\}$ and $P=\left\{p \mid T_{p} \neq 0\right\}$. Let " $y \in$ $\left[x_{1}, \ldots, x_{n}\right]$ " be an abbreviation for

$$
\bigvee\left\{m y=\sum_{i=1} k_{i} x_{i}: 0 \neq m \in \omega ; k_{1}, \ldots, k_{n} \in \omega\right\}
$$

(i.e., $y$ is in the pure subgroup generated by $x_{1}, \ldots, x_{n}$ ). Let $\varphi_{T}$ be the conjunction of the following sentences: for each $p \in S$ the sentence

$$
\widehat{\Lambda} \forall x_{1} \ldots x_{n} \forall y\left(y \in\left[x_{1}, \ldots, x_{n}\right] \vee \bigvee_{m} \forall z\left(p^{m_{z}}-y \notin\left[x_{1}, \ldots, x_{n}\right]\right)\right)
$$

and the following sentence if $P$ is infinite:

$$
\bigwedge_{n} \forall x_{1} \ldots x_{n} \forall y\left(y \in\left[x_{1}, \ldots, x_{n}\right] \vee \underset{p \in P}{\bigvee} \forall z\left(p z-y \notin\left[x_{1}, \ldots, x_{n}\right]\right)\right) \text {. }
$$

Then it is easy to see that $\varphi_{T}$ is true in $G$ if and only if $G$ is $T$-admissible.

It is well known that the $T$-admissibility of $G$ is not sufficient to imply $\operatorname{Ext}(G, T)=0$ when $G$ is uncountable. Under the assumption $\mathrm{V}=\mathrm{L}$ we obtain necessary and sufficient conditions for $\operatorname{Ext}(G, T)=0$ when $G$ has cardinality $N_{1}$.

2.3 TheOREM $(\mathrm{V}=\mathrm{L})$. Let $T$ be a reduced torsion group and let $G$ be a torsion-free group of cardinality $\aleph_{1}$. Then $\operatorname{Ext}(G, T)=0$ if and only if there is an $\aleph_{1}-$ filtration $\Delta$ of $G$ satisfying

(1) $\Delta(0)$ is $T$-admissible;

and for all $\nu<\kappa$ :

(2) $\bar{\Delta}(\nu)$ is $T$-admissible.

Proof. If $\overline{\bar{T}}<\aleph_{1}$, Theorem 2.3 is an immediate consequence of Theorems 1.2, 1.5 and 2.1. In the case when $\overline{\bar{T}}$ is arbitrary, the sufficiency of the given condition for $\operatorname{Ext}(G, T)=0$ is clear from Theorems 1.2 and 2.1. As for necessity, notice first that we may assume that $T$ is a direct sum of cyclic groups; this is because if $B$ is a basic subgroup of $T$, then for any torsion-free group $C, \operatorname{Ext}(C, T)=0$ if and only if $\operatorname{Ext}(C, B)=0$ (see Saisada [21]). Then by definition of $T$-admissible, there is a countable direct summand $T_{1}$ of $T$ such that for any $C, C$ is $T$-admissible if and only if $C$ is $T_{1}$-admissible. (Simply choose $T_{1}$ so that $T_{p} \neq 0$ iff $\left(T_{1}\right)_{p} \neq 0$ and $T_{p}$ is unbounded iff $\left(T_{1}\right)_{p}$ is unbounded.) Thus if $T=T_{1} \oplus T_{2}, \operatorname{Ext}(G, T)=\operatorname{Ext}\left(G, T_{1}\right) \oplus \operatorname{Ext}\left(G, T_{2}\right)$. If there is no $\kappa_{1}$-filtration $\Delta$ of $G$ satisfying (1) and (2) then by Theorems 1.5 and 2.1 and the choice of $T_{1}, \operatorname{Ext}\left(G, T_{1}\right) \neq 0$ and hence $\operatorname{Ext}(G, T) \neq 0$. 
The above theorem provides a solution, under the assumption of the Axiom of Constructibility, to Problem 80 of [10] for the case when $\overline{\bar{G}}=\aleph_{1}$. By induction then we may obtain a criterion for the vanishing of $\operatorname{Ext}(G, T)$ when $\bar{G}=\kappa_{n}$ for $1<n<\omega$ by making use of Theorem 1.7 to reduce the problem to considering groups of cardinality $<\aleph_{n-1}$. Since the theorems of $\S 1$ do not deal with the case of the vanishing of $\operatorname{Ext}(C, A)$ when $\bar{C}$ is singular, we do not have, at the present time, a complete criterion for the vanishing of $\operatorname{Ext}(G, T)$ when $\overline{\bar{G}}>$ * $_{\omega}$.

We shall show in the next section that Theorem 2.3 is not a theorem of ZFC; more precisely, the existence of a filtration satisfying (2) is not a necessary condition for $\operatorname{Ext}(G, T)=0$ in all models of ZFC.

The criterion of Theorem 2.3 is obviously much more complicated than that of Theorem 2.1. The next result reinforces the conclusion that no simpler criterion is provable in ZFC; more precisely, the analog of Corollary 2.2 is not a theorem of $\mathrm{ZFC}$ for uncountable $G$.

2.4 THEOREM $(\mathrm{V}=\mathrm{L})$. Let $T$ be an unbounded reduced torsion group. (i) If $\mathrm{k}$ is a regular uncountable cardinal $>\overline{\bar{T}}$ which is not weakly compact, then there does not exist a sentence $\varphi$ of $L_{\infty \times}$ with the property that for all torsion-free groups $G$ of cardinality $\kappa, \operatorname{Ext}(G, T)=0$ if and only if $G$ satisfies $\varphi$. (ii) There does not exist a sentence $\varphi$ of $L_{\infty}$ with the property that for all torsion-free groups $G, \operatorname{Ext}(G, T)=0$ if and only if $G$ satisfies $\varphi$.

Proof. (i) It suffices to prove that there are torsion-free groups $G_{1}$ and $G_{2}$ of cardinality $\kappa$ such that $\operatorname{Ext}\left(G_{1}, T\right)=0, \operatorname{Ext}\left(G_{2}, T\right) \neq 0$ but $G_{1} \equiv_{\infty \times} G_{2}$, i.e., $G_{1}$ and $G_{2}$ satisfy the same sentences of $L_{\infty \infty}$. For $G_{1}$ we take the free group of cardinality $\kappa$; so clearly $\operatorname{Ext}\left(G_{1}, T\right)=0$. By a theorem of Gregory (see [12]: proof unpublished) there is a nonfree group $G_{2}$ of cardinality $\kappa$ such that $G_{1} \equiv_{\infty \kappa} G_{2}$. (Gregory's theorem requires that $\kappa$ be nonweakly compact since it uses Theorem 0.3.) A proof of Gregory's theorem is outlined in [7, Theorem 3.5]. An examination of the construction given there shows that $G_{2}$ may be constructed so that there is a $\kappa$-filtration $\Delta$ of $G_{2}$ such that for every $\nu<\mu<\kappa, \Delta(\mu) / \Delta(\nu+1)$ is free, but for every limit ordinal $\lambda$ of cofinality $\omega$, $\Delta(\lambda+1) / \Delta(\lambda) \cong Q$ (the rationals). Since $T$ is a reduced and unbounded torsion group, $T$ is not cotorsion i.e., $\operatorname{Ext}(Q, T) \neq 0$ (cf. [9, Corollary 54.4]). Hence $\{\lambda<\kappa \mid \operatorname{Ext}(\Delta(\lambda), T) \neq 0\}$ is stationary in $\kappa$ since it contains the set of all limit ordinals $<\kappa$ of cofinality $\omega$. Therefore by Theorem 1.5, $\operatorname{Ext}\left(G_{2}, T\right)$ $\neq 0$.

As for part (ii), it suffices to prove that for every $\kappa$ there are torsion-free groups $G_{1}$ and $G_{2}$ such that $\operatorname{Ext}\left(G_{1}, T\right)=0, \operatorname{Ext}\left(G_{2}, T\right) \neq 0$ but $G_{1} \equiv_{\infty \times} G_{2}$. But this follows from part (i) and the fact that there are arbitrarily large 
regular nonweakly-compact cardinals (since successor cardinals are not weakly compact).

In contrast to Theorem 2.4 we have the following theorem of ZFC.

2.5 THEOREM. Let $A$ be a group of cardinality $<\kappa$, where $\kappa$ is weakly compact (resp. strongly compact). There is a sentence $\varphi$ of $L_{\infty \times}$ with the property that for any group $C$ with $\bar{C}<\kappa$ (resp. $\bar{C}$ arbitrary), $\operatorname{Ext}(C, A)=0$ if and only if $C$ satisfies $\varphi$.

Proof. Let $\mathcal{G}$ be the set of all groups $G$ of cardinality $<\kappa$ such that $\operatorname{Ext}(G, T)=0$. Let $\varphi$ be the sentence

$$
\bigwedge_{\lambda<\kappa} \forall\left\{\nu_{\mu}: \mu<\lambda\right\} \underset{G \in \mathcal{G}}{\bigvee_{\mathcal{G}}}\left(\left\langle\left\{\nu_{\mu}: \mu<\lambda\right\}\right\rangle \cong G\right) \text {. }
$$

It is not hard to see that $\varphi$ is (equivalent to) a sentence of $L_{\infty \times}$. Clearly $\varphi$ has the property that $C$ satisfies $\varphi$ if and only if every subgroup of $C$ of cardinality $<\kappa$ is in $\mathcal{G}$. The proof is completed by applying Theorem 1.6.

2.6 COROLLARY. Let $\mathrm{k}$ be a strongly compact cardinal and let $A$ be a group of cardinality $<\kappa$. The class of all groups $C$ such that $\operatorname{Ext}(C, A)=0$ is definable in $L_{\infty \times}$.

2.7 REMARK. By a result of Solovay (personal communication) there exists (if the existence of a supercompact cardinal is consistent) a model of ZFC in which the first strongly compact cardinal $\kappa$ has the property that there are arbitrarily large regular $\lambda<\kappa$ such that there is a stationary set $E \subseteq \lambda$ consisting of ordinals of cofinality $\omega$ and such that $E \cap \mu$ is not stationary in $\mu$ for any $\mu<\lambda$. It follows from the techniques in [7] and from the above results that in this model $\kappa$ is the first cardinal $\lambda$ such that (for any fixed noncotorsion $A$ of small cardinality), the class of groups $C$ satisfying $\operatorname{Ext}(C, A)=0$ is definable in $L_{\infty \lambda}$. Thus in some sense 2.6 is a best possible result.

3. The vanishing of Ext and Martin's Axiom. We shall consider the vanishing of Ext under the assumption of Martin's Axiom and prove that Ext may vanish even though the conditions of Theorem 1.5 are not satisfied. However, under the assumption of Martin's Axiom we are not able to determine general criteria for the vanishing of Ext. We shall be considering torsion-free groups which are homogeneous, i.e., every nonzero element is of the same type (see $[10, \S 85])$. If $A$ is a group let us say that a type $t$ is $A$-admissible if the (unique) rank one torsion-free group of type $t, R(t)$, satisfies $\operatorname{Ext}(R(t), A)=0$. If $A$ is a torsion group, Theorem 2.1 implies that the $T$-admissible types form an initial segment of the lattice of types, which is proper if and only if $T$ is not cotorsion. By Lemma 1.1, if $\operatorname{Ext}(G, A)=0$ then the type of every nonzero element of $G$ is $A$-admissible. Recall that $A$ is cotorsion iff $\operatorname{Ext}(Q, A)=0$, 
where $Q$ is the group of rationals under addition. (See $[9, \S 54]$.)

3.1 TheOREM (MA $+\sim \mathrm{CH}$ ). Let $A$ be a countable group which is not cotorsion and let $t$ be a type which is $A$-admissible. There is a torsion-free group $C$ of cardinality $\kappa_{1}$ which is homogeneous of type $t$ such that $\operatorname{Ext}(C, A)=0$ but $C$ does not have an $\aleph_{1}$-filtration satisfying (1) and (2) of Theorem 1.5.

Theorem 3.1 will be a consequence of Theorem 3.2. First, recall that a torsion-free group $G$ is $\kappa$-separable if every subset of $G$ of cardinality $<\kappa$ is contained in a completely decomposable summand of $G$ of cardinality $<\kappa$. Let us call a torsion-free group $G$ weakly $\kappa$-separable if every subset of $G$ of cardinality $<\kappa$ is contained in a completely decomposable $\kappa$-pure subgroup of $G$ of cardinality $<\kappa$. (Recall that a subgroup $H$ of $G$ is completely decomposable if it is a direct sum of rank one torsion-free groups [10, §86]. Also, $H$ is $\kappa$-pure in $G$ if $H$ is a direct summand of every $G^{\prime}$ such that $H \subseteq G^{\prime} \subseteq G$ and $G^{\prime} / H$ has cardinality $<\kappa[9, \$ 31]$.)

3.2 THEOREM (MA $+\sim \mathrm{CH}$ ). Let $A$ be a countable group. Let $G$ be a weakly $\kappa_{1}$-separable group of cardinality $<2^{\kappa_{0}}$ which is homogeneous of type $t$. Then $\operatorname{Ext}(G, A)=0$ if and only if $t$ is $A$-admissible.

Before proving Theorem 3.2 let us derive Theorem 3.1.

Proof of 3.1. Let $C$ be the union of a smooth chain $\left\{C_{\nu} \mid \nu<x_{1}\right\}$ of countable completely decomposable groups of type $t$ such that for every successor ordinal $\nu+1<N_{1}, C_{\nu+1}$ is a direct summand of $C_{\mu}$ whenever $\nu+1<\mu<\aleph_{1}$, and such that for every limit ordinal $\sigma<\aleph_{1}, C_{\sigma+1} / C_{\sigma}$ is isomorphic to $Q$ (the rationals). The construction of $C$ can be carried out as in [7] where the case of type $t=(0,0, \ldots, 0, \ldots)$ is done explicitly. (The proof uses 3.4 below. See also [14] for the case $t=(0,0, \ldots)$.) By construction, $C$ is obviously weakly $\kappa_{1}$-separable, hence by Theorem $3.2, \operatorname{Ext}(C, A)=$ 0 . But if $\Delta$ is the $\kappa_{1}$-filtration of $C$ given by $\Delta(\nu)=C_{\nu}$, the set $S=\{\nu<$ $\left.\aleph_{1} \mid \operatorname{Ext}(\bar{\Delta}(\nu), A) \neq 0\right\}$ equals the set of limit ordinals $\left\langle\aleph_{1}\right.$ and thus is stationary in $\aleph_{1}$. Therefore Lemma 1.8 implies that $C$ does not have an $\aleph_{1}$-filtration satisfying (1) and (2) of Theorem 1.5.

Before proving 3.2, let us recall two important results about homogeneous completely decomposable groups. The proofs are to be found in Fuchs [10, 86.6 and 86.8].

3.4 Lemma. Let $G$ be a completely decomposable homogeneous group. Then

(i) every pure subgroup of $G$ is completely decomposable; and

(ii) if $G$ is of finite rank, then every pure subgroup of $G$ is a direct summand.

Proof of 3.2. Let $t$ be an $A$-admissible type. Consider a short exact sequence 


$$
0 \rightarrow A \stackrel{\bullet}{\rightarrow} B \stackrel{\pi}{\rightarrow} G \rightarrow 0 \text {. }
$$

Let $P$ be the set of all homomorphisms $f: H \rightarrow B$ such that $H$ is a pure subgroup of $G$ of finite rank and $\pi f=1_{H}$. If we prove that $P$ satisfies conditions (0.5.1), (0.5.2), and (0.5.3), then by Theorem 0.5 there is a function $g: G \rightarrow B$ such that for every finite subset $X$ of $G, g$ restricted to $X$ is contained in a member of $P$. It follows easily that $g$ is a homomorphism such that $\pi f=1_{G}$ i.e., (3.2.1) splits.

Condition (0.5.1) is obvious. As for (0.5.2), given $a \in A$, and $f: H \rightarrow B$ in $P$ let $H^{\prime}$ be the pure subgroup of $G$ generated by $H$ and $a$. By hypothesis, $H^{\prime}$ is contained in a completely decomposable group. Hence by Lemma 3.4(i), $H^{\prime}$ is completely decomposable. Since $H^{\prime}$ is also of finite rank and homogeneous, Lemma 3.4(ii) implies that $H$ is a direct summand of $H^{\prime}$. It then follows easily that $f$ extends to a homomorphism $f^{\prime}: H^{\prime} \rightarrow B$ in $P$.

Thus it remains to verify (0.5.3). Given an uncountable subset $P^{\prime}$ of $P$ we assert

There is an uncountable subset $\tilde{P}$ of $P^{\prime}$ and a pure subgroup $\tilde{G}$ of $G$ such that $\tilde{G}$ is completely decomposable and every element of $\tilde{P}$ has its domain contained in $\tilde{G}$.

Before proving the assertion, let us see how to use it to conclude the proof of (0.5.3).

Let $\tilde{P}$ and $\tilde{G}$ be as in (3.2.2). Let us write $\tilde{G}=\bigoplus_{i \in I} R_{i}$ where each $R_{i}$ is a rank one group of type $t$. Let $\tilde{P}=\left\{f_{\nu} \mid \nu<N_{1}\right\}$ where $f_{\nu}: H_{\nu} \rightarrow B$. By the argument used to prove (0.5.2) we may extend each $f_{v}$ to an element of $P$ with domain of the form $\bigoplus_{i \in I_{p}} R_{i}$ for some finite subset $I_{v}$ of $I$. Hence we may assume that each $H_{v}$ is of this form. Moreover, since a countable union of countable sets is countable, we may assume (replacing $\tilde{P}$ by an uncountable subset) that there is an $n$ such that $I_{v}$ has $n$ elements for each $\nu<\aleph_{1}$. Then there is a subset $J$ of $I$ which is maximal with respect to the property that $J$ is contained in $I_{v}$ for uncountably many $\nu$. Since $A$ is countable, there are only countably many functions on $\bigoplus_{i \in J} R_{i}$ which belong to $P$ (since $f \in P$ implies $\pi f=1_{\text {dom }(f)}$ ). Hence we may assume (again replacing $\tilde{P}$ by an uncountable subset) that for any $\mu, \nu<\kappa_{1}, J \subseteq I_{\nu} \cap I_{\mu}$ and $f_{\nu}\left|\bigoplus_{i \in J} R_{i}=f_{\mu}\right| \bigoplus_{i \in J} R_{i}$. Now by the maximality of $J$, if $i \in I_{0}-J$ there are only countably many $\nu$ such that $i \in I_{\nu}$. Hence there exists $\nu \neq 0$ such that $I_{0} \cap I_{\nu}=J$. Let $H=$ $\bigoplus\left\{R_{i}: i \in I_{0} \cup I_{\nu}\right\}$. Define a function $g: H \rightarrow B$ by $g\left|\bigoplus_{i \in I_{0}} R=f_{0}, g\right|$ $\bigoplus_{i \in I_{v}} R_{i}=f_{v}$. Then $g$ is a well-defined homomorphism which is an element of $P$. Thus (0.5.3) is verified, assuming, of course, that (3.2.2) holds.

It remains therefore to prove (3.2.2). Suppose $P^{\prime}=\left\{f_{\nu} \mid \nu<\aleph_{1}\right\}$ where $f_{v}: H_{\nu} \rightarrow B$. As before we may assume (replacing $P^{\prime}$ by an uncountable subset) that there is a pure subgroup $G_{0}$ of $G$ such that $G_{0}$ is maximal with 
respect to the property that $G_{0}$ is a pure subgroup of uncountably many $H_{v}$. By Lemma 3.4 (ii) $G_{0}$ is a direct summand of $H_{\nu}$; let us write $H_{\nu}=G_{0} \oplus H_{\nu}^{\prime}$.

We shall construct by transfinite induction a smooth increasing chain $\left\{G_{\nu} \mid \nu<\aleph_{1}\right\}$ of pure completely-decomposable subgroups of $G$ and a strictly increasing sequence of ordinals $\left\{\tau_{\nu+1} \mid \nu<N_{1}\right\}$ such that $H_{\tau_{p+1}} \subseteq G_{\nu+1}$. Moreover, the chain $\left\{G_{\nu} \mid \nu<\aleph_{1}\right\}$ will be constructed so that $G_{\nu}$ is a direct summand of $G_{\nu+1}$ for all $\nu$. Thus $\tilde{G}=\cup_{\nu<\kappa_{1}} G_{\nu}$ and $\tilde{P}=\left\{f_{\tau_{\nu+1}} \mid \nu<\kappa_{1}\right\}$ will satisfy (3.2.2).

Suppose $\left\{G_{\nu} \mid \nu<\mu\right\}$ and $\left\{\tau_{\nu} \mid \nu<\mu\right\}$ have been defined. If $\mu$ is a limit ordinal let $G_{\mu}=\cup_{v<\mu} G_{v}$. If $\mu=\nu+1$ let $C$ be a countable $\kappa_{1}$-pure subgroup of $G$ which contains $G_{v}$. There exists $\tau_{\nu+1}>\tau_{\rho+1}$ for all $\rho<\nu$ such that $H_{\tau_{v+1}}^{\prime} \cap C=0$. (If not, then since $C$ is countable, there would exist an element $c \in C$ and uncountably many $\tau<N_{1}$ such that $c \in H_{\tau}^{\prime}$; but then the pure closure of $G_{0} \cup\{c\}$ contradicts the maximality of $G_{0}$.) Let $G_{\mu}$ be the pure closure of $G_{\nu}+H_{\tau_{\nu+1}}^{\prime}$. We claim that $G_{\nu}$ is a direct summand of $G_{\mu}$. Consider the canonical map $\varphi: G_{\mu} / G_{\nu} \rightarrow G / C$. By construction, $\varphi$ is injective. Thus $G_{\mu} / G_{v}$ is isomorphic to $\varphi\left(G_{\mu} / G_{\nu}\right)$. Now by choice of $C$, and the fact that $G$ is weakly $\kappa_{1}$-separable, $\varphi\left(G_{\mu} / G_{\nu}\right)$ is contained in a (countable) completely decomposable subgroup $D$ of $G / C$ which is homogeneous of type $t$. Thus every nonzero element of $\varphi\left(G_{\mu} / G_{v}\right)$ has type $<t$ (with respect to $\left.\varphi\left(G_{\mu} / G_{\nu}\right)\right)$. On the other hand, since homomorphisms increase type, every nonzero element of $\varphi\left(G_{\mu} / G_{\nu}\right)$ has type $\geqslant t$. Thus $\varphi\left(G_{\mu} / G_{\nu}\right)$ is homogeneous of type $t$. By Theorem 86.6 of [10], $\varphi\left(G_{\mu} / G_{\nu}\right)$ is completely decomposable. Thus $G_{\mu} / G_{\nu}$ is completely decomposable and homogeneous of type $t$ and it then follows from Proposition 86.5 of [10] that $G_{\nu}$ is a direct summand of $G_{\mu}$.

3.5 Remarks. (1) The verification of (0.5.3) is somewhat simplified in case the type of $G=(0,0, \ldots, 0, \ldots)$. In this case $\varphi\left(G_{\mu} / G_{v}\right)$ is a subgroup of a free group and is therefore free; hence $G_{\nu}$ is a direct summand of $G_{\mu}$.

(2) Note that the homogeneity of $G$ is made use of in Lemma 3.4 and in the use of 86.5 and 86.6 from Fuchs [10]. We do not know if any analog of Theorem 3.2 holds if the homogeneity assumption is dropped.

The methods of Theorem 3.2 may be used to prove the following.

3.6 THEOREM (MA $+\sim \mathrm{CH})$. Let $\Lambda$ be a countable ring of left global dimension < 1 . Let $A$ be a countable $\Lambda$-module and let $M$ be a $\Lambda$-module of cardinality $<2^{\mathrm{N}_{0}}$ such that every countable submodule of $M$ is contained in a free direct summand of $M$. Then $\operatorname{Ext}_{\Lambda}^{1}(M, A)=0$.

4. Independence results. We shall give here a series of results which show that certain problems are not solvable in ZFC. In particular we show that different answers to the problem are obtained in models of $\mathrm{ZFC}+\mathrm{V}=\mathrm{L}$ 
and $\mathrm{ZFC}+\mathrm{MA}+\sim \mathrm{CH}$. We begin with the result of Shelah which is the inspiration for this paper.

4.1 TheOREM (Shelah). (i) $(\mathrm{V}=\mathrm{L})$ If $G$ is a group such that $\operatorname{Ext}(G, Z)=0$, then $G$ is free.

(ii) (MA $+\sim \mathrm{CH})$ For any cardinal $\kappa>\aleph_{1}$ there is a nonfree group $G$ of cardinality $\kappa$ such that $\operatorname{Ext}(G, Z)=0$.

Proof. (i) The proof is by induction on the cardinality of $G$. For countable $G$ the result is a classical theorem of ZFC_([25]; see also [10, \$99]). If (i) has been proved for cardinals $<\overline{\bar{G}}$ and $\kappa=\overline{\bar{G}}$ is regular, the result follows from Theorem 1.7 and the inductive hypothesis since $G$ has a $\kappa$-filtration such that for all $\nu<\kappa, \Delta(\nu)$ and $\bar{\Delta}(\nu)$ are free. (In particular we obtain the result for groups of cardinality $\kappa_{n}, n<\omega$.) If $\kappa$ is singular the result follows from the following theorem of Shelah [23] (proved for cardinals of cofinality $N_{0}$ and $\kappa_{1}$ by P. Hill).

4.2 THeOREM (SHeLAH). If $\kappa$ is a singular cardinal and $G$ is a group of cardinality $\kappa$ such that every subgroup of $G$ of cardinality less than $\kappa$ is free, then $G$ is free.

We continue the proof of 4.1: (ii) By Theorem 3.1 there is a group $C$ of cardinality $\kappa_{1}$ which is not free but $\operatorname{Ext}(C, Z)=0$. Given $\kappa>\kappa_{1}$ let $G=C^{(\kappa)}$ (i.e., the direct sum of $\kappa$ copies of $C$ ). Then $G$ is certainly not free, but $\operatorname{Ext}(G, Z)=\Pi_{\kappa} \operatorname{Ext}(C, Z)=0$.

A group $G$ is $\aleph_{1}$-coseparable if every countable subgroup of $G$ is free and every subgroup $H$ such that $G / H$ is countable contains a direct summand $K$ such that $G / K$ is countable [14, p. 133]. Griffith proved that $G$ is $\kappa_{1}$-coseparable if and only if $\operatorname{Ext}\left(G, Z^{(\omega)}\right)=0$ [14, Theorem 1.91]. The following result is then proved exactly as the above theorem.

4.3 THEOREM. (i) $(\mathrm{V}=\mathrm{L})$ If $G$ is $\aleph_{1}$-coseparable, then $G$ is free.

(ii) (MA $+\sim \mathrm{CH})$ For any cardinal $\kappa \geqslant \kappa_{1}$, there is an $\kappa_{1}$-coseparable group $G$ of cardinality $k$ which is not free.

Let us call $G$ a Griffith group if $G$ is a nonfree $\kappa_{1}$-separable subgroup of $Z^{\omega}$ of cardinality $\kappa_{1}$ defined as in Griffith [14, Theorem 14]. (There may be more than one Griffith group, depending on the choice of the sequences $\sigma_{\lambda}(n)$ in Griffith's construction.) Note that the construction of such a group is carried out in ZFC so that it makes sense to consider properties of Griffith groups in any model of ZFC. We shall see that whether $\operatorname{Ext}(G, A)$ vanishes is not always decidable in ZFC. (Compare this with Theorem 2.4.)

4.4 THEOREM. (i) $(\mathrm{V}=\mathrm{L})$ If $G$ is a Griffith group and $A$ is a countable group which is not cotorsion, then $\operatorname{Ext}(G, A) \neq 0$. 
(ii) (MA $+\sim \mathrm{CH})$ If $G$ is a Griffith group and $A$ is any countable group, then $\operatorname{Ext}(G, A)=0$.

Proof. (i) An examination of the construction in [14] shows that if $G$ is a Griffith group then $G$ has an $\aleph_{1}$-filtration $\Delta$ such that for every limit ordinal $\lambda, \bar{\Delta}(\lambda)$ has nontrivial divisible part. (Referring to the notation of Griffith's proof, $\Delta(\beta)=G_{\beta}$ for every $\beta<\aleph_{1}$.) Therefore $\{\nu: \operatorname{Ext}(\bar{\Delta}(\lambda), A) \neq 0\}$ is the set of all limit ordinals $\left\langle\aleph_{1}\right.$ since $A$ is not cotursion. Hence by Lemma 1.4, $\operatorname{Ext}(G, A) \neq 0$.

(ii) This is an immediate consequence of Theorem 3.2 since the type $t=(0,0, \ldots, 0, \ldots)$ is obviously $A$-admissible for any $A$.

Note that 4.4(ii) shows that it is consistent with ZFC that there is no countable $A$ such that $\operatorname{Ext}(C, A)=0$ implies $C$ is free.

A group $G$ is defined to be totally $\kappa_{1}$-separable if $G$ is $\kappa_{1}$-free and every subgroup of $G$ is $\aleph_{1}$-separable (cf. [14, p. 133]). Griffith asks whether every $\kappa_{1}$-separable group is totally $\kappa_{1}$-separable. (Here we use $\kappa_{1}$-separable as Griffith does to include the hypothesis $\kappa_{1}$-free.)

4.5 TheOREM. (i) $(\mathrm{V}=\mathrm{L})$ There is an $\kappa_{1}$-separable group of cardinality $\kappa_{1}$ which is not totally $\kappa_{1}$-separable.

(ii) (MA $+\sim \mathrm{CH})$ Every $\kappa_{1}$-separable group of cardinality $\kappa_{1}$ is totally $\kappa_{1}$-separable.

Proof. Griffith proved that if $G$ is $\kappa_{1}$-separable then $G$ is totally $\kappa_{1}$-separable if and only if $\operatorname{Ext}\left(G, Z^{(\omega)}\right)=0$ [14, Theorems 191, 193, 194]. Thus, since $Z^{(\omega)}$ is not cotorsion, the theorem is an immediate consequence of Theorem 4.4 (i) and Theorem 3.2.

Call $G$ a Baer group if $\operatorname{Ext}(G, T)=0$ for all torsion groups $T$. Call $G$ a $\kappa$-Baer group if $\operatorname{Ext}(G, T)=0$ for all torsion groups of cardinality $<\kappa$. An $\aleph_{0}$-Baer group is necessarily torsion-free (by [9, 53(D)]). Griffith proved that every Baer group is free ([13] and [14]). In fact, his proof shows that for any $G$, if $\kappa=\overline{\bar{G}}^{+}$(the successor cardinal of $\overline{\bar{G}}$ ) and $G$ is a $\kappa$-Baer group, then $G$ is free (cf. [10, Exercise 2, p. 193]). This is the best that can be proved in ZFC, at least for $\kappa=\kappa_{1}$, as we see from the following result.

4.6 Theorem. (i) $(\mathrm{V}=\mathrm{L})$ For any group $G$, if $G$ is an $\aleph_{1}$-Baer group, then $G$ is free.

(ii) $(\mathrm{MA}+\sim \mathrm{CH})$ For any cardinal $\kappa>\kappa_{1}$, there is an $\kappa_{1}$-Baer group of cardinality $\mathrm{\kappa}$ which is not free.

Proof. (i) Let $T$ be the direct sum of $\kappa_{0}$ copies of each finite cyclic group. We prove by induction on $\kappa$ that if $\overline{\bar{G}}=\kappa$ and $\operatorname{Ext}(G, T)=0$ then $G$ is free. If $\kappa<\kappa_{0}$, this follows from Griffith's result (and is provable in ZFC). If $\kappa$ is a 
regular cardinal $\geqslant \kappa_{1}$ then Theorem 1.5 and the inductive hypothesis imply that $G$ has a $\kappa$-filtration $\Delta$ such that for all $\nu<\kappa, \Delta(\nu)$ and $\bar{\Delta}(\nu)$ are free. Hence $G$ is free. If $\kappa$ is singular, the inductive hypothesis and Lemma 1.1 imply that every subgroup of $G$ of cardinality $<\kappa$ is free. Hence by $4: 2 G$ is free.

(ii) Let $G$ be a Griffith group. Then by 4.4 (ii), $G$ is an $\kappa_{1}$-Baer group which is not free. If $\kappa>\kappa_{1}$ then $G^{(\kappa)}$, the direct sum of $\kappa$ copies of $G$, is an $\kappa_{1}$-Baer group of cardinality $\kappa$ which is not free.

4.7 Open problem. Is it relatively consistent with ZFC that $\kappa_{1}$-coseparable does not imply totally $\aleph_{1}$-separable?

\section{REFERENCES}

1. R. Baer, The subgroup of the elements of finite order of an abelian group, Ann. of Math. (2) 37 (1936), 766-781.

2. W. Boos, Infinitary compactness without strong inaccessibility, J. Symbolic Logic 41 (1976), 33-38.

3. H. Cartan and S. Eilenberg, Homological algebra, Princeton Univ. Press, Princeton, N. J. 1956. MR 17, 1040.

4. M. Dickman, Large infinitary languages, North-Holland, Amsterdam, 1975.

5. F. Drake, Set theory, an introduction to large cardinals, North-Holland, Amsterdam, 1974.

6. P. C. Eklof, Infinitary equivalence of abelian groups, Fund. Math. 81 (1974), 305-314. MR 50 \#6829.

7. MR 52 \#599.

8. L. Fuchs, Abelian groups, Akad. Kiadó, Budapest, 1958; Pergamon Press, New York, 1960. MR 21 \#5672; 22 \#2644.

9. , Infinite abelian groups, Vol. I, Academic Press, New York and London, 1970. MR 41 \#333.

10. , Infinite abelian groups, Vol. II, Academic Press, New York and London, 1973. MR 50 \#2362.

11. K. Gödel, The consistency of the continuum hypothesis, Princeton Univ. Press, Princeton, N. J., 1940. MR 2, 66.

12. J. Gregory, Abelian groups infinitcrily equivalent to free ones, Notices Amer. Math. Soc. 20 (1973), A-500. Abstract \#73T-E77.

13. P. A. Griffith, A solution to the splitting mixed group problem of Baer, Trans. Amer. Math. Soc. 139 (1969), 261-269. MR 39 \#317.

14. Infinite abelian group theory, Univ. of Chicago Press, Chicago, 1970. MR 44 \#6826.

15. R. Jensen, The fine structure of the constructible hierarchy, Ann. Math. Logic 4 (1972), 229-308; erratum, ibid. 4 (1972), 443. MR 46 \#8834.

16. H. J. Keisler, Model theory for infinitary logic, North-Holland, Amsterdam, 1971. MR 49 \#8855.

17. K. Kunen, Indescribability and the continuum, Proc. Sympos. Pure Math., vol. 13, part I, Amer. Math. Soc., Providence, R. I., 1971, pp. 199-203. MR 44 \#63.

18. A. Mekler, Applications of logic to group theory, Ph. D. Dissertation, Stanford Univ., 1976.

19. _ The number of $k$-free groups and the size of Ext (to appear).

20. J. Rotman, Shelah's solution of Whitehead's problem (typescript).

21. E. Saisada, An application of Kulikov's basic subgroups in the theory of abelian mixed groups, Bull. Acad. Polon. Sci. 4 (1956), 411-413. 
22. S. Shelah, Infinite abelian groups, Whitehead problem and some constructions, Israel J. Math. 18 (1974), 243-256. MR 50 \# 9582.

23. and transversals, Israel J. Math. 21 (1975), 319-349.

24. R. M. Solovay and S. Tennenbaum, Iterated Cohen extensions and Souslin's problem, Ann. of Math. (2) 94 (1971), 201-245. MR 45 \#3212.

25. K. Stein, Analytische Funktionen mehrerer komplexer Veränderlichen zu vorgegebenen Periodizitätsmoduln und das zweite Cousinsche Problem, Math. Ann. 123 (1951), 201-222. MR 13, 224.

Department of Mathematics, University of California, Irvine, California 92717 